\title{
Physics of outflows near solar active regions
}

\author{
D. J. Price and Y. Taroyan \\ Aberystwyth University, Physical Sciences Building, Aberystwyth, SY23 3BZ, UK \\ Correspondence to: D. J. Price (djp12@aber.ac.uk)
}

Received: 15 August 2014 - Revised: 9 December 2014 - Accepted: 10 December 2014 - Published: 7 January 2015

\begin{abstract}
Hinode/EIS observations have revealed outflows near active regions which remain unexplained. An outflow region observed by the EUV Imaging Spectrometer (EIS) that appears slightly redshifted at low temperatures and blueshifted at higher temperatures is presented. We conduct simulations and use those to create synthetic line profiles in order to replicate the observed line profiles of an apparent open structure. The results of the forward modelling support a scenario whereby long loops consisting of multiple strands undergo a cyclical process of heating and cooling on timescales of approximately $80 \mathrm{~min}$.
\end{abstract}

Keywords. Solar physics astrophysics and astronomy (corona and transition region; ultraviolet emissions)

\section{Introduction}

The EUV Imaging Spectrometer (EIS; Culhane et al., 2007) has given us spectacular spectral images of various phenomena since its launch. Of particular interest are outflow regions that may help to explain some of the Sun's remaining mysteries. Such outflow regions are usually located at the periphery of many solar active regions. Large amounts of material leaving the Sun is always of interest in terms of the coronal heating problem, and in terms of the origins of the solar wind.

It has been established that transition region emission lines display no net Doppler shifts or slight redshifts (Doyle et al., 2002), which are often observed together with blueshifted higher-temperature lines. This has been reported in a number of situations: for example, Warren et al. (2011) found redshifted cold loops adjacent to a high-temperature blueshifted outflow region.

In the following work we investigate a particular outflow region that has been noted in several other papers (e.g. McIntosh and De Pontieu, 2009; Warren et al., 2011). We approx- imate what appears to be an open structure as a long loop and perform hydrodynamic simulations to replicate the observed emission line profiles, allowing us to suggest physical parameters such as temperature and density. We create synthetic line profiles and compare them to the observed profiles to determine the accuracy of our simulations and to establish the physical nature of the outflows.

\section{Observations}

For this study, we concentrated on an active region visible on 20 February 2007. Hinode/EIS observations of the active region were carried out using the 1" wide slit in raster mode. In the following analysis, we select the Fe VIII $185.21 \AA$, Fe X 184.54 $\AA$, and Fe XII 195.12 ̊ spectral lines with formation temperatures of $\log T \approx 5.6,6.0$, and 6.2.

EIS level 0 data files are processed with the eis_prep software, using the default options. The eis_wave_corr procedure and the level 1 data are used to calculate the wavelength corrections for each spectral line. We fit a single Gaussian profile to each line spectrum, using the eis_auto_fit software. The reference wavelength of each line profile has been updated to match the rest wavelength found in a laboratory, and is taken from Warren et al. (2011). EIS Doppler velocities need calibrating against a reference wavelength, where the quiet Sun velocity averages to zero. For this work the Fe VIII $185.21 \AA$ line is chosen as a reference. The other lines are calculated relative to the final wavelength of this line.

The intensity maps in Fig. 1 show fan-like structures at the top left corners of the images that become bright mainly in the low-temperature line of Fe VIII 185.21 ̊. The connectivity of the fan-like structures is unclear in the small field of view of EIS. These are either long loops or open structures that extend into the solar wind.

We selected three pixels from the outflow region as shown in Fig. 1 and plotted their line profiles, interpolating between 


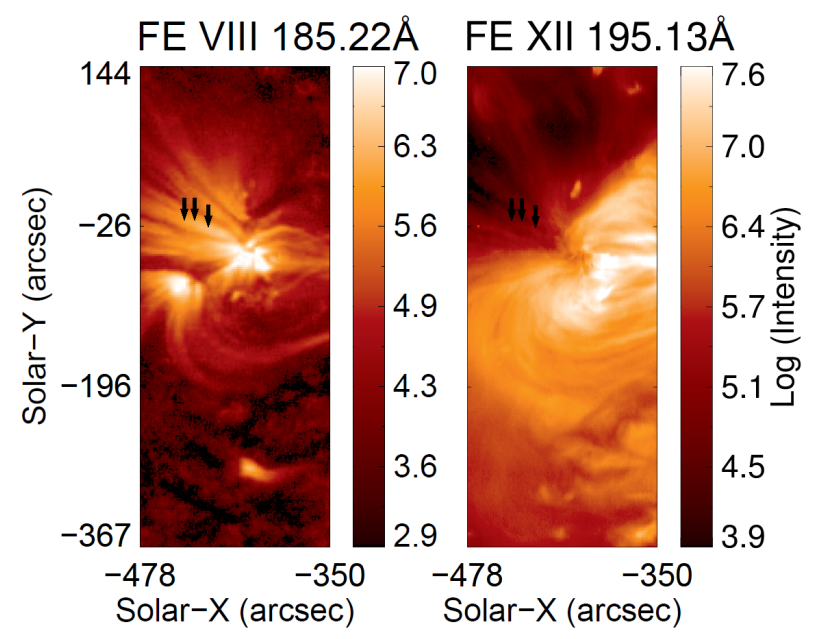

Figure 1. Intensity of the Fe VIII and Fe XII emission lines for the observed area; the outflow region is visible flowing towards the upper left part of the images. The black arrows indicate the approximate locations of the pixels used for the line profiles of Fig. 2. In order from left to right they correspond to the dashed line, the dotted line, and the solid line respectively (as drawn in Fig. 2).

the available data points to complete the curves (Fig. 2). Although the three sets of line profiles are not identical, they have similar intensity despite being taken from different spatial locations. This was also true for other pixels in the region which are not shown. The Doppler shifts for the pixels show slight redshifts for Fe VIII, barring the dotted centre one, which is slightly blue, and blueshifts for Fe X and Fe XII.

\section{Modelling}

We approximated the observed structure as a long loop and modelled it using the 1-D hydrodynamics and radiation code HYDRAD (Bradshaw and Cargill, 2013). The simulations were carried out taking into account the 15 most abundant elements in the solar atmosphere; to begin with they were considered to be in equilibrium for calculating the initial state and then not in equilibrium for the ensuing simulations. This ability of the code combined with its adaptive grid allows us to get an accurate picture of how all of the ions are affected throughout the simulations.

The loop was taken to be $100 \mathrm{Mm}$ long, from a visual estimate, including $2 \mathrm{Mm}$ of chromosphere at each end. These footpoints of the loop were held at a constant temperature of $2 \times 10^{4} \mathrm{~K}$, and their density varied to find the best value. The initial apex temperature of the loop was determined by the code from the footpoint density, so it varied accordingly. The initial state of the loop is found by uniform time-independent background heating that persists throughout the simulation to prevent it from cooling to collapse.

With this loop we then attempted to recreate the observed line profiles by injecting a single time-dependent heating

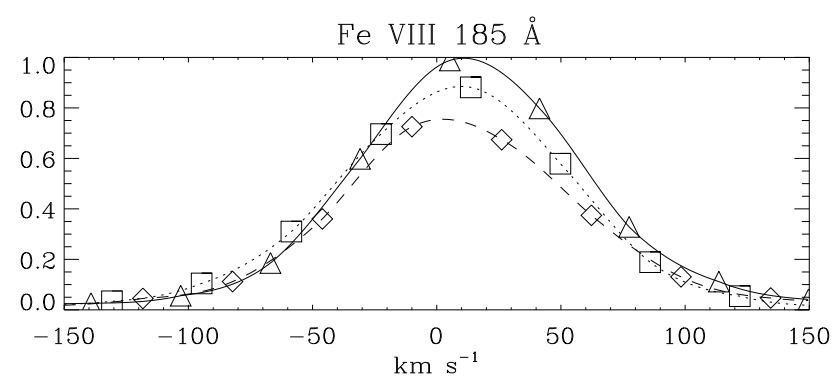

Fe X $184.5 \AA$
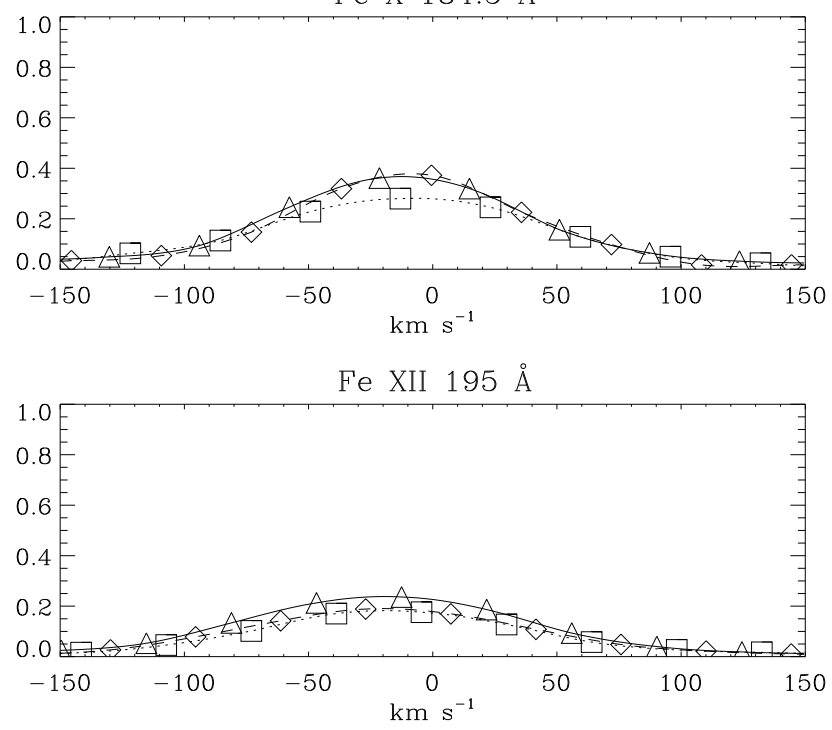

Figure 2. Observed line profiles for three pixels chosen from the outflow region seen in Fig. 1. The different line styles distinguish the different pixels and the symbols indicate the corresponding observed data points; the gaps are filled via interpolation. The top profile is for the $185 \AA$ Fe VIII $\left(4 \times 10^{5} \mathrm{~K}\right)$ emission line, the middle profile is for the $184 \AA \mathrm{Fe} \mathrm{x}\left(1 \times 10^{6} \mathrm{~K}\right)$ line, and the bottom profile is for the $195 \AA \mathrm{Fe}$ XII $\left(1.3 \times 10^{6} \mathrm{~K}\right)$ line. This ordering of lines is consistent throughout the paper.

pulse into the top of the chromosphere at the first footpoint. The parameters of the pulse were varied over a number of simulations, but its temporal evolution always took the form of a linear increase from zero to maximum heating, a plateau, and then a linear decrease back to zero.

The simulations began immediately with the injection of the pulse and continued until the loop had settled back almost to its initial state. The physical and ion data were then forward-modelled using a separate part of the code in order to synthesize the line profiles of interest in the same way as described by Taroyan et al. (2006).

The profiles correspond to the emission of the loop from 18.7 to $20.0 \mathrm{Mm}$, which corresponds to the 1" slit of EIS when transformed out of the semicircular loop coordinate frame. We construct the profiles for a single loop, 10 superimposed threads, and 100 superimposed threads. In the latter two cases there is a random time lag between each two, oth- 

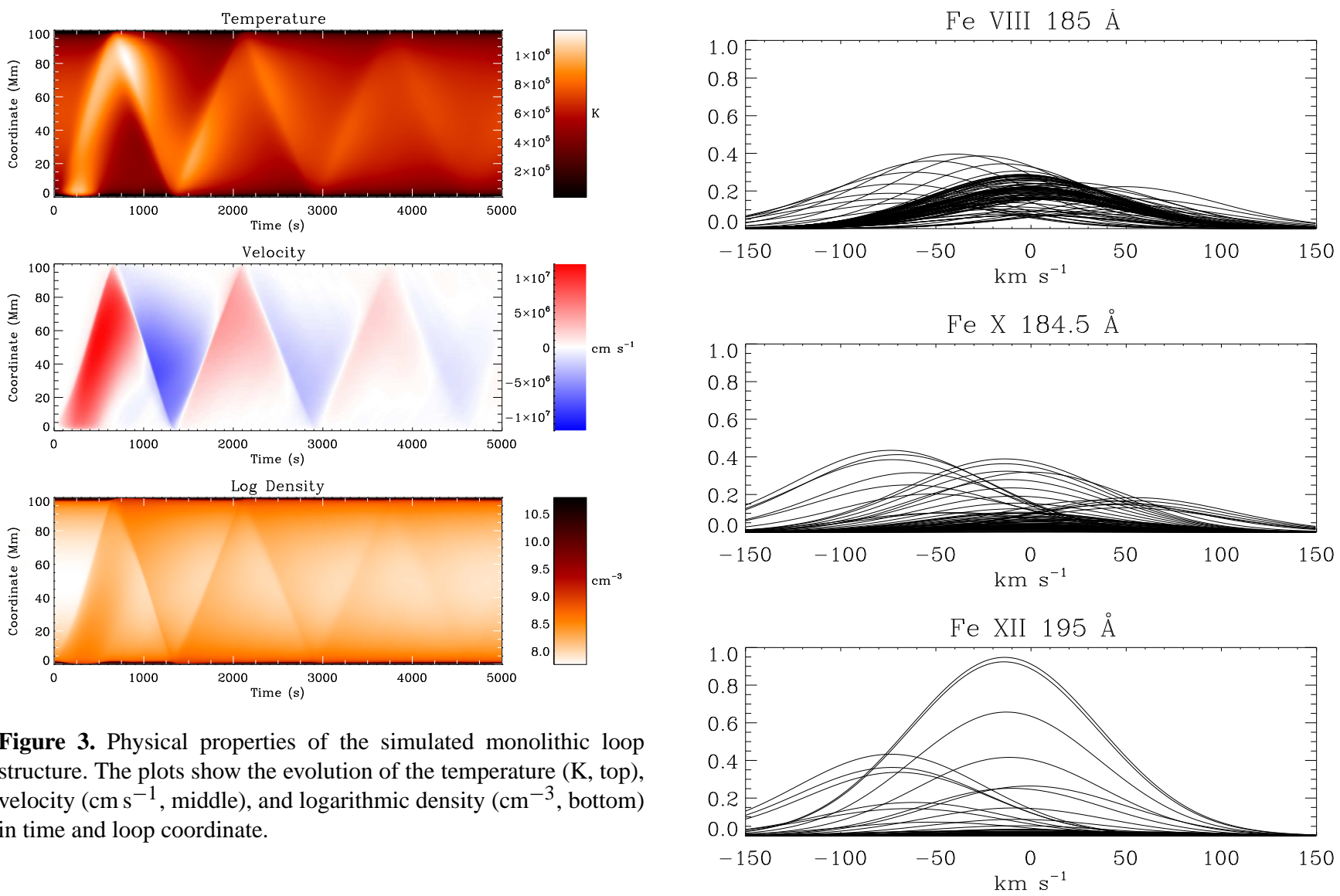

erwise identical, neighbouring threads as in the modelling of Taroyan and Bradshaw (2014). The line profiles, superimposed or not, are then fitted with a single Gaussian to determine their Doppler shifts for comparison to the observed values.

\section{Results and discussion}

It was found that the observations were reproduced most accurately by a loop with an initial density of $7 \times 10^{9} \mathrm{~cm}^{-3}$ which had a corresponding apex temperature of approximately $0.7 \mathrm{MK}$. The pulse consisted of a volumetric heating rate of $6 \times 10^{-3} \mathrm{erg} \mathrm{cm}^{-3} \mathrm{~s}^{-1}$, a scale height of $2 \mathrm{Mm}$, and a total duration of $500 \mathrm{~s}$. This was broken into $200 \mathrm{~s}$ for the linear increase, $100 \mathrm{~s}$ for the plateau, and $200 \mathrm{~s}$ for the linear decrease. The loop reached a maximum temperature of 1.1 MK as it was heated. It was found that heating to the same peak temperature but with a lower heating rate over a longer period of time resulted in lower blueshifts.

The physical properties of the simulated loop are shown in Fig. 3. The effects of the heating injection are reflected by all three plots, including multiple rebounds at the footpoints as the loop undergoes a single cycle of heating and cooling to initial low temperatures. We may be able to obtain a better fit in future with a dedicated open-structure model.

Figure 4. Synthetic line profiles, from a $5000 \mathrm{~s}$ simulation, for a loop consisting of 1 strand. There is a $50 \mathrm{~s}$ interval between each plotted line.

First, we inspect the single-thread case (Fig. 4): we find bad agreement with the observations; none of the three profiles are persistently Doppler shifted one way or the other; and where they are shifted, the values exceed those observed. Also, the profile peaks are not consistent with the observations. The superposition of 10 threads across the line of sight as shown in Fig. 5 is a clear improvement, with some periods of time being fairly comparable to the observations; however there is still no consistency in shifts amongst the emission lines. The 100-thread case (Fig. 6) yields further improvements, with the emission lines being fairly consistent with each other over time, and their peak heights are comparable to those observed.

The Gaussian fitting in the 100-thread case, taken forward as the most accurate representation of the observations, revealed Doppler shifts of approximately $0,-10$, and $-25 \mathrm{~km} \mathrm{~s}^{-1}$ for the Fe VIII, Fe X, and Fe XII emission lines respectively. These values are fairly consistent with the observations considering the $\pm 5 \mathrm{~km} \mathrm{~s}^{-1}$ instrument error (Culhane et al., 2007). 

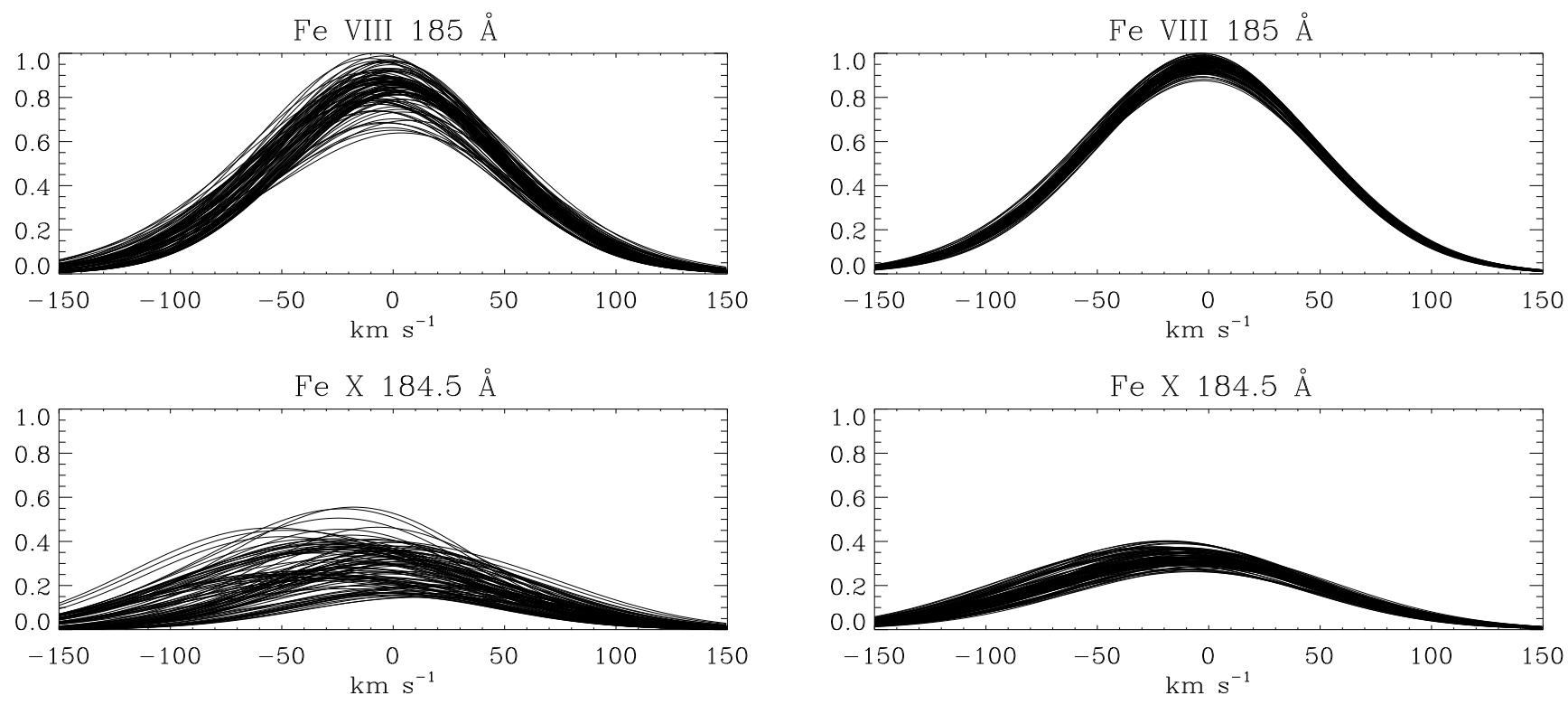

Fe XII $195 \AA$

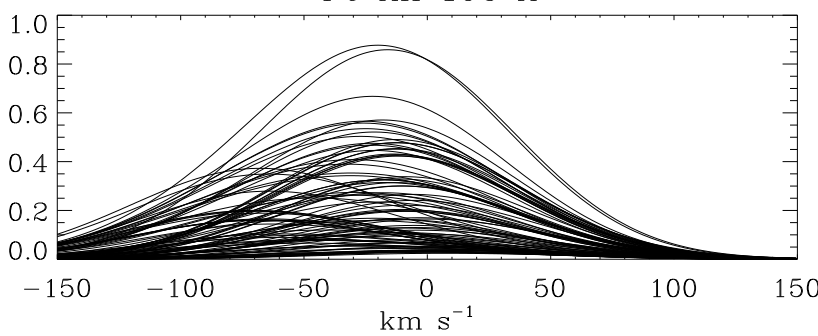

Figure 5. Synthetic line profiles, from a $5000 \mathrm{~s}$ simulation, for a loop consisting of 10 strands. There is a $50 \mathrm{~s}$ interval between each plotted line.

\section{Conclusions}

We find that the observed structure can be approximated well by an impulsively heated multi-thread loop $100 \mathrm{Mm}$ long. We determine the footpoint density to be $7 \times 10^{9} \mathrm{~cm}^{-3}$. The superposition of multiple threads, each individually heated by a single pulse, results in the higher-temperature $\mathrm{Fe} X$ and Fe XII lines being persistently blueshifted throughout the simulation time, whereas the shifts corresponding to the cooler Fe VIII line tend towards zero for an increasing number of threads. The good agreement between the observed and simulated line profiles allows us to interpret the physical nature of the outflows and the relatively low intensities at higher temperatures. We conclude that there are at least 100 threads along the line of sight. Each thread undergoes a cyclical process of heating to about $1 \mathrm{MK}$ followed by a cooling to about $0.7 \mathrm{MK}$ on a timescale of approximately $80 \mathrm{~min}$. While having each thread only heated once over the simulation time is a strong assumption, if we were to have instances of multiple heating in some threads and no heating in other threads our results would cease to match the observations. The con-

Acknowledgements. We wish to thank S. Bradshaw for providing Price is supported by the STFC (UK). Hinode is a Japanese mission developed and launched by ISAS/JAXA, with NAOJ as domestic partner and NASA and UKSA as international partners. It is operated by these agencies in co-operation with ESA and NSC (Norway).

Topical Editor L. Ofman thanks D. Banerjee and the anonymous referee for their help in evaluating this paper.

Figure 6. Synthetic line profiles, from a $5000 \mathrm{~s}$ simulation, for a loop consisting of 100 strands. There is a $50 \mathrm{~s}$ interval between each plotted line.

sequences of more general random pulses will be addressed in a future study. 


\section{References}

Bradshaw, S. J. and Cargill, P. J.: The Influence of Numerical Resolution on Coronal Density in Hydrodynamic Models of Impulsive Heating, Astrophys. J., 770, 12, doi:10.1088/0004637X/770/1/12, 2013.

Culhane, J. L., Harra, L. K., James, A. M., Al-Janabi, K., Bradley, L. J., Chaudry, R. A., Rees, K., Tandy, J. A., Thomas, P., Whillock, M. C. R., Winter, B., Doschek, G. A., Korendyke, C. M., Brown, C. M., Myers, S., Mariska, J., Seely, J., Lang, J., Kent, B. J., Shaughnessy, B. M., Young, P. R., Simnett, G. M., Castelli, C. M., Mahmoud, S., Mapson-Menard, H., Probyn, B. J., Thomas, R. J., Davila, J., Dere, K., Windt, D., Shea, J., Hagood, R., Moye, R., Hara, H., Watanabe, T., Matsuzaki, K., Kosugi, T., Hansteen, V., and Wikstol, Ø.: The EUV Imaging Spectrometer for Hinode, Solar Phys., 243, 19-61, 2007.
Doyle, J. G., Madjarska, M. S., Roussev, I., Teriaca, L., and Giannikakis, J.: Temporal variability in the Doppler-shift of solar transition region lines, Astron. Astrophys., 396, 255-267, 2002.

McIntosh, S. W. and De Pontieu, B.: Observing Episodic Coronal Heating Events Rooted in Chromospheric Activity, Astrophys. J. Lett., 706, L80-L85, 2009.

Taroyan, Y. and Bradshaw, S. J.: Forward-Modeling of Doppler Shifts in EUV Spectral Lines, Solar Phys., 289, 1959-1970, 2014.

Taroyan, Y., Bradshaw, S. J., and Doyle, J. G.: Nanoflare heating of coronal loops: hydrodynamic response and observational consequences, Astron. Astrophys., 446, 315-325, 2006.

Warren, H. P., Ugarte-Urra, I., Young, P. R., and Stenborg, G.: The Temperature Dependence of Solar Active Region Outflows, Astrophys. J., 727, 58, doi:10.1088/0004-637X/727/1/58, 2011. 